\title{
COMPUTATIONAL SIMULATIONS OF FORE AND AFT RADIATION FROM DUCTED FANS
}

\author{
V. Ahuja ${ }^{\dagger}$ \\ Combustion Research and Flow \\ Technology, Inc. \\ Dublin, PA 18917 USA
}

\author{
Y. Ozyoruk ${ }^{\dagger \dagger}$ \\ Middle East Technical University \\ Ankara, Turkey 06531
}

\author{
L. Long ${ }^{\S}$ \\ Pennsylvania State University \\ University Park, PA 16802
}

\begin{abstract}
In this paper, we extend the work of Ozyoruk and Long, for predicting farfield noise from ducted fans using a higher order coupled Euler/Navier-Stokes solver along with a Kirchhoff formulation, to full engine configurations on distributed parallel computers. Incorporating the exhaust along with the inlet raises many issues relating to appropriate grid topologies, multigrid acceleration and the unsteady numerics. These issues are addressed in this paper and simulations pertaining to a realistic engine geometry are presented. Qualitative comparisons are in good agreement with results from a frequency domain code, pertaining to radiation from a dipole in a cylinder. Simulations relating to radiation from an engine geometry, and with and without the centerbody, with and without mass flow are also shown.
\end{abstract}

\subsection{INTRODUCTION}

In the past fifty years environmental awareness, increased sensitivity to noise, and protests by communities directly in the flight path, near airports, have placed huge demands on reduction of noise generated by commercial and transport aircraft. Since the main source of noise is engine-related noise, the quest for quieter skies has led to a major focus on reducing engine noise without sacrificing engine thrust and performance. Consequently engine technology has undergone a major transformation. Engines used forty years ago incorporated turbojet technology, where the major source of sound was mixing noise attributed to the jet exhaust mixing with the surrounding air. Mixing noise was reduced with the advent of low-bypass-ratio turbofan engines, since the fan was used to augment thrust and the core and fan exhausts were mixed internally before being vented. This has been further reinforced with the introduction of the high bypass ratio engines wherein a significant portion of the thrust is provided by the fan, thereby further leading to a reduction of jet exhaust velocities. As a consequence, the emphasis on noise reduction has now shifted to fan-related noise and the acoustics of the ducted fan system. Tyler and Sofrin [1] have investigated and classified noise generating mechanisms in axial flow systems as those due to the rotating blades, the interaction between the rotor and the

\footnotetext{
${ }^{\dagger}$ Research Scientist, Member AIAA.

I† Associate Professor, Department of Aeronautical Engineering, Member AIAA.

$\S$ Professor, Department of Aerospace Engineering, Associate Fellow AIAA.
}

Copyright $(\odot 2000$ by the authors. Published by AIAA with permission. stator and the interaction of the boundary layer and inlet disturbances with the rotor blades. The fundamental tonal noise generating mechanism is attributed to the rotating pressure patterns or spinning modes by the rotor or the fan at the blade passage frequency or its harmonics. This is a direct consequence of blade loading and blade thickness. Also important is the interaction of rotor wakes cutting through the stator blades producing noise at multiples of the blade passage frequency. As the fan size is increased, with increasing bypass ratio engines, rotor-stator interactions play a more significant role as a noise generating mechanism. Recent advances in parallel computing have facilitated the simulation of entire blade rows of stators and rotors [2]. This has provided the ability to capture the circumferential variation in flow features such as non-uniform wakes interacting with stator vanes and the rotating modes that are responsible for noise [3].

For the purpose of design and reduction of noise, it becomes critical to efficiently capture the far-field acoustic field that is produced in the engine. However, in high bypass ratio engines, the BPF tones propagate inside the engine nacelle, and aft of the stator blade row through the exhaust duct before being radiated out as sound. The $\mathrm{BPF}$ tones radiate depending on whether the frequency is above or below the critical value based on the characteristics of the noise generating mechanisms, the flow conditions in the duct and other characteristics of the duct. Furthermore, the shape of the nacelle can lead to significant refraction making transmission through the duct complicated. The downstream radiation is further supplemented by the noise due to mixing in the shear layer at the aft end. Most of existing methods utilized in the prediction of radiation and transmission make simplifying assumptions that preclude capturing of the complex flow and wave propagation phenomena inherent in such systems, such as nonlinear effects, aerodynamic acoustic coupling and wave refraction. Eversman et al. [4] use a combination of the finite element and wave envelope techniques with a velocity potential based formulation. Although, the method is efficient at high frequencies, it fails to account for aerodynamic-acoustic coupling and nonlinear effects that might influence the wave propagation process. Furthermore, in the case of aft radiation a predetermined function has to be specified to account for the shape of the shear layer. Boundary element based formulations used by Myers [5], Dunn [12] have proved to be very robust and provide keen insight into ducted fan noise. However, ???? utility is limited to simplified flows with a uniform mean flow, where the flow does not considerably influence the acoustic field. 
In this paper, we propose to solve the radiation/transmission problem from first principles by numerically solving the Euler/Navier-Stokes equations in the vicinity of the entire engine utilizing a spatially higher order time-accurate method. Our method has been adequately proven to predict sound for the JT15D engine inlet in the past [6] and has compared favorably with semi-analytical methods in predicting the BPF tones. The evolution of parallel distributed computing makes permissible the simultaneous investigation of the forward and aft radiation arcs of an engine with fine spatial resolution. An efficient implementation of the moving surface Kirchhoff formula of Farassat and Myers [7] permits the prediction of far-field noise $[6,8,10]$. In this paper, noise generation mechanisms are analytically specified as source terms that mimic the superposition of multiple modes and harmonics related to the blade passage frequency. It must be noted that our scheme is robust [8] and unlike most CAA schemes can be readily used to solve problems involving realistic geometries and flow conditions. Furthermore, our method can be easily incorporated in the design cycle for enhancing nacelle design, engine ducting, analysis of cut-off modes and can be readily extended to a comprehensive Navier-Stokes solver that could be used to analyze noise generating mechanisms such as rotor-stator blade rows, turbulence/boundary layer interactions and shear layers.

The code used by Ozyoruk and Long [6, 8, 10] was written in connection machine Fortran (CMF), which is very similar to Fortran 90 and High Performance Fortran (HPF). The current code is written in Fortran 90 and Message Passing Interface (MPI). The new MPI code is very complicated compared to the CMF code. It is a real shame that HPF has not caught on, and people have to use MPI.

In the next section, we provide details of the numerical model including specification of the source terms and implementation of multigrid acceleration that is critical to obtaining a steady state solution devoid of numerical noise. In section 3 , we discuss results of simulations of duct transmission and radiation from a generic engine and the validation of our method against boundary element solutions for radiation of a dipole in a cylinder. The paper ends with concluding remarks and recommendations for future work.

\subsection{NUMERICAL MODEL}

\subsection{Interior Equations: Navier Stokes/Euler}

The 3-D time dependent Navier-Stokes/Euler equations are solved in body fitted generalized coordinates in the interior of the computational domain, with the nonreflecting boundary conditions solved on the outer boundaries. The time dependent partial differential equations representing the far field conditions are expressed in compatible form, so that the differential flux operator is transparent across the entire computational domain. For the sake of completeness, the equations are written here in conservative generalized coordinates.

$$
\frac{\partial \hat{Q}}{\partial t}+\frac{\partial\left(\hat{F}-\hat{F}_{v}\right)}{\partial \xi}+\frac{\partial\left(\hat{G}-\hat{G}_{v}\right)}{\partial \eta}+\frac{\partial\left(\hat{H}-\hat{H}_{v}\right)}{\partial \zeta}=0
$$

where $\mathrm{Q}$ is the vector of conservative state variables and $\mathrm{F}, \mathrm{G}$ and $\mathrm{H}$ are the flux vectors. Fv, $\mathrm{Gv}$ and $\mathrm{Hv}$ are the standard viscous fluxes.

$$
\begin{aligned}
& \hat{\mathrm{Q}}=\frac{\mathrm{Q}}{\mathrm{J}} \\
& Q=\left[\begin{array}{c}
\rho \\
\rho u \\
\rho v \\
\rho w \\
e
\end{array}\right] \\
& \hat{\mathrm{F}}=\frac{1}{\mathrm{~J}}\left[\begin{array}{c}
\rho \mathrm{U} \\
\rho \mathrm{Uu}+\xi_{\mathrm{x}} \mathrm{p} \\
\rho \mathrm{UV}+\xi_{\mathrm{y}} \mathrm{p} \\
\rho \mathrm{Uw}+\xi_{\mathrm{z}} \mathrm{p} \\
(\mathrm{e}+\mathrm{p}) \mathrm{U}-\xi_{\mathrm{t}} \mathrm{p}
\end{array}\right], \hat{\mathrm{G}}=\frac{1}{\mathrm{~J}}\left[\begin{array}{c}
\rho \mathrm{V} \\
\rho \mathrm{Vu}+\eta_{\mathrm{x}} \mathrm{p} \\
\rho \mathrm{Vv}+\eta_{\mathrm{y}} \mathrm{p} \\
\rho \mathrm{Vw}+\eta_{\mathrm{z}} \mathrm{p} \\
(\mathrm{e}+\mathrm{p}) \mathrm{V}-\eta_{\mathrm{t}} \mathrm{p}
\end{array}\right] \text {, } \\
& \hat{H}=\frac{1}{J}\left[\begin{array}{c}
\rho W \\
\rho W u+\zeta_{x} p \\
\rho W v+\zeta_{y} p \\
\rho W w+\zeta_{z} p \\
(e+p) W-\zeta_{t} p
\end{array}\right]
\end{aligned}
$$

The contravariant velocity components are written as

$$
\begin{aligned}
& \mathrm{U}=\xi_{\mathrm{x}} \mathrm{u}+\xi_{\mathrm{y}} \mathrm{v}+\xi_{\mathrm{z}} \mathrm{w}+\xi_{\mathrm{t}} \\
& \mathrm{v}=\eta_{\mathrm{x}} \mathrm{u}+\eta_{\mathrm{y}} \mathrm{v}+\eta_{\mathrm{z}} \mathrm{w}+\eta_{\mathrm{t}} \\
& \mathrm{W}=\zeta_{\mathrm{x}} \mathrm{u}+\zeta_{\mathrm{y}} \mathrm{v}+\zeta_{\mathrm{z}} \mathrm{w}+\zeta_{\mathrm{t}}
\end{aligned}
$$

and pressure is computed from the conservative variables by the relation.

$$
p=(\gamma-1)\left[e-\rho \frac{\left(u^{2}+v^{2}+w^{2}\right)}{2}\right]
$$

The spatial derivatives are computed using a fourth order stencil in each coordinate direction. Time 
integration is performed using a four stage Runge-Kutta method with a blend of Jameson's [9] second and fourth order type artificial dissipation, for damping the spurious high wavenumber components. The scheme is stable for CFL numbers less than $2 \sqrt{2}$. However, for wave propagation type problems CFL numbers of less than 1 are preferred. This is mainly because the amplification factor is close to 1 for the entire range of wavenumber components at CFL numbers less than 1. However, the phase error can be significant for the higher wavenumber components and it limits the resolution of the scheme to 12 cells per wavelength.

\subsection{Multigrid Convergence Acceleration}

Since the emphasis in our approach is to utilize the full Euler/Navier-stokes equations, all problems regarding sound radiation presuppose an established steady state flow solution. Once, a steady state solution has been obtained, the sources responsible for generation of sound are turned on and the wave propagation process is superposed on the steady state solution. This places a very stringent requirement of a smooth steady state solution devoid of spurious waves. Unfortunately, the very characteristics of low dispersion and dissipation, that make the scheme suitable to simulation of wave propagation processes, hinder convergence to steady state. The convergence problem is alleviated in our method through the use of multigrid acceleration, [10] wherein the low frequency numerical modes are aliased as high frequency modes on successive grids of varying coarseness. This is particularly attractive in acoustic problems with a mean flow, because of the high resolution nature of the grid that is needed to resolve acoustic frequencies of interest. However, in the case of complex geometries, especially those with multiple corner points, trailing edges etc., the coarse grids need to be generated carefully, in order to retain these defining features of the geometries that are critical to the flow. This has been illustrated in figures (1a) and (1b) where the fine base grid and the coarsest level grid are shown retaining critical features of the geometry. For example, the trailing edge, beginning of the centerbody, the aft end of the nozzle, and the corner points at the junction of the axis and the farfield are maintained in the coarse grids as constraining parameters in much the same manner as they appear in the finest grid. In our implementation we have used Jameson's full approximation scheme [11] with a 3 level V-cycle formulation. Convergence of the solution on each successive coarse grid is driven by the residual on the finer grid with the help of a forcing function that reflects the difference between the convective $\rho$ and dissipative fluxes $D$ at the two grid levels.

$$
\begin{aligned}
& P_{2 h}=W_{h}^{2 h}\left(F_{h}\left(Q_{h}\right)-D_{h}\left(Q_{h}\right)\right. \\
& -\left(F_{2 h}\left(Q_{2 h}^{(0)}\right)-D_{2 h}\left(Q_{2 h}^{(0)}\right)\right.
\end{aligned}
$$

Here the subscripts $h$ stand for the fine grid and $2 \mathrm{~h}$ for the next level of coarse grids and the superscript represents the initial Runge Kutta stage. The integration process is performed on the next grid level as follows:

$$
\begin{gathered}
\mathrm{Q}_{2 \mathrm{~h}}^{(0)}=\mathrm{T}_{2 \mathrm{~h}}^{(0)} \mathrm{Q}_{\mathrm{h}} \\
\mathrm{Q}_{2 \mathrm{~h}}^{(1)}=\mathrm{Q}_{2 \mathrm{~h}}^{(0)}-\frac{1}{4}(\mathrm{~J} \Delta \mathrm{t})_{2 \mathrm{~h}}\left[\mathrm{~F}\left(\mathrm{Q}_{2 \mathrm{~h}}^{(0)}\right)-\mathrm{D}\left(\mathrm{Q}_{2 \mathrm{~h}}^{(0)}\right)+\mathrm{P}_{2 \mathrm{~h}}\right] \\
\mathrm{Q}_{2 \mathrm{~h}}^{(2)}=\mathrm{Q}_{2 \mathrm{~h}}^{(0)}-\frac{1}{3}(\mathrm{~J} \Delta \mathrm{t})_{2 \mathrm{~h}}\left[\mathrm{~F}\left(\mathrm{Q}_{2 \mathrm{~h}}^{(1)}\right)-\mathrm{D}\left(\mathrm{Q}_{2 \mathrm{~h}}^{(0)}\right)+\mathrm{P}_{2 \mathrm{~h}}\right] \\
\mathrm{Q}_{2 \mathrm{~h}}^{(3)}=\mathrm{Q}_{2 \mathrm{~h}}^{(0)}-\frac{1}{3}(\mathrm{~J} \Delta \mathrm{t})_{2 \mathrm{~h}}\left[\mathrm{~F}\left(\mathrm{Q}_{2 \mathrm{~h}}^{(2)}\right)-\mathrm{D}\left(\mathrm{Q}_{2 \mathrm{~h}}^{(0)}\right)+\mathrm{P}_{2 \mathrm{~h}}\right] \\
\mathrm{Q}_{2 \mathrm{~h}}^{(4)}=\mathrm{Q}_{2 \mathrm{~h}}^{(0)}-\frac{1}{3}(\mathrm{~J} \Delta \mathrm{t})_{2 \mathrm{~h}}\left[\mathrm{~F}\left(\mathrm{Q}_{2 \mathrm{~h}}^{(3)}\right)-\mathrm{D}\left(\mathrm{Q}_{2 \mathrm{~h}}^{(0)}\right)+\mathrm{P}_{2 \mathrm{~h}}\right]
\end{gathered}
$$

The procedure is continued until the coarsest mesh is reached. A correction to the solution is then performed on the coarsest mesh

$$
\Delta \mathrm{Q}_{\mathrm{vh}}=\mathrm{Q}_{\mathrm{vh}}^{(4)}-\mathrm{Q}_{\mathrm{vh}}^{(0)}
$$

And the accumulated correction is passed back successively until the finest is reached.

$$
\begin{array}{r}
\Delta \mathrm{Q}_{\mathrm{rh} / 2}=\mathrm{Q}_{\mathrm{rh} / 2}^{\mathrm{new}}-\mathrm{Q}_{\mathrm{rh} / 2}=\mathrm{I}_{\mathrm{rh}}^{\mathrm{rh} / 2}\left(\mathrm{Q}_{\mathrm{rh}}^{\text {new }}-\mathrm{Q}_{\mathrm{rh}}^{(0)}\right) \\
\mathrm{Q}_{\mathrm{h}}^{\mathrm{n}+1}=\mathrm{Q}_{\mathrm{h}}^{\text {new }}=\mathrm{Q}_{\mathrm{h}}+\Delta \mathrm{Q}_{\mathrm{h}}
\end{array}
$$

Restriction of data $\mathrm{T}_{\mathrm{nh}}$, onto coarser grids is accomplished through direct injection, whereas prolongation $\mathrm{I}_{\mathrm{rh}}^{\mathrm{rh} / 2}$ is performed by bilinear interpolation.

Needless to say, including the multigrid procedure in the solution algorithm has resulted in better overall accuracy for the steady state solution, and in turn for the acoustic pressure levels. Multigrid acceleration has also resulted in tremendous savings in computational resources, as is seen in Figure 2, which is a convergence comparison for single grid and multigrid convergence characteristics for a converging-diverging nozzle type flow, that is indicative of wide range of flow regimes in terms of Mach Number. The computational savings due to acceleration in convergence of the multigrid solution far outweigh the added expense of the three-level iterative multigrid method over a single level computation.

\subsection{Kirchhoff Formulation:}

Limitations on computational resources preclude the extension of the computational domain into the farfield. The acoustic response in the near-field is bridged by means of the Kirchhoff formula [15] of Farassat and Myers [7] to the far-field. This formulation of the Kirchhoff integration surface is for arbitrarily moving and 
deforming surfaces and is coupled with our Euler-Navier Stokes solver.

On simpler geometries, the Kirchhoff surface can be conveniently constructed along constant surfaces defined by $\xi, \eta$ or $\zeta$ grid lines, since the surface parameters, such as normal vector and surface area are known apriori. In more complicated geometries, combinations of grid lines can be utilized to construct a closed surface, with the given exception that this surface will now have piecewise discontinuous normal vectors and surface areas. In such cases, the individual surface elements are tagged and an additional data structure of pointers is stored.

For a nondeforming Kirchhoff surface that is in rectilinear motion, the Kirchhoff formula for the acoustic pressure $\mathrm{p}^{\prime}$ at the observer location $x$ and observer time $t$ takes the following form:

$$
4 \pi \mathrm{p}^{\prime}(\mathrm{x}, \mathrm{t})=\iint_{\mathrm{s}}\left[\frac{\mathrm{E}_{1}}{\mathrm{R}\left(1-\mathrm{M}_{\mathrm{R}}\right)}+\frac{\mathrm{p}^{\prime} \mathrm{E}_{2}}{\mathrm{R}^{2}\left(1-\mathrm{M}_{\mathrm{R}}\right)}\right]_{\tau^{*}} \mathrm{dS}
$$

where

$$
\mathrm{R}=|\mathrm{V}|, \stackrel{\mathrm{v}}{\mathrm{R}}=\stackrel{\mathrm{V}}{\mathrm{X}}-\mathrm{V} \mathrm{y}(\tau), \mathrm{M}_{\mathrm{R}}=\stackrel{\mathrm{v}}{\mathrm{M}} \cdot \stackrel{\mathrm{v}}{\mathrm{R}} / \mathrm{R},
$$

and

$$
\begin{gathered}
\mathrm{E}_{1}=-\hat{\mathrm{n}} \cdot \nabla \mathrm{p}^{\prime}+(\stackrel{\mathrm{v}}{\mathrm{M}} \cdot \hat{\mathrm{n}})\left(\stackrel{\mathrm{V}}{\mathrm{M}} \cdot \nabla \mathrm{p}^{\prime}\right) \\
+ \\
{\left[\frac{\cos \vartheta-\mathrm{V} \cdot \hat{\mathrm{n}}}{\mathrm{c}_{\infty}\left(1-\mathrm{M}_{\mathrm{R}}\right)}-\frac{\mathrm{M} \cdot \hat{\mathrm{n}}}{\mathrm{c}_{\infty}}\right] \frac{\partial \mathrm{p}^{\prime}}{\partial \tau}} \\
\mathrm{E}_{2}=\frac{1-\mathrm{M}^{2}}{\left(1-\mathrm{M}_{\mathrm{R}}\right)^{2}} \cos \vartheta-\hat{\mathrm{M}} \cdot \hat{\mathrm{n}}
\end{gathered}
$$

The Kirchhoff surface and the observer are seen to travel at $-\mathrm{M}_{\infty}$, and the elapsed time for a signal to travel from a Kirchhoff surface element to the observer will always be the same, thereby, ostensibly simplifying the numerical coupling between the flow solver and the Kirchhoff method. Thus, the emission time for a Kirchhoff surface element $e$ is given by

$$
\tau_{\mathrm{e}}^{*}=\mathrm{t}-\mathrm{R}_{\mathrm{e}} / \mathrm{c}_{\infty}
$$

where

$$
\mathrm{R}_{\mathrm{e}}=\frac{-\left(\overline{\mathrm{x}}_{1}-\overline{\mathrm{y}}_{1}\right) \mathrm{M}_{\infty}+\overline{\mathrm{R}}}{\beta^{2}}
$$

with $\beta^{2}=1-\mathrm{M}_{\infty}^{2}$ and

$$
\overline{\mathrm{R}}=\sqrt{\left(\overline{\mathrm{x}}_{1}-\overline{\mathrm{y}}_{1}\right)^{2}+\beta^{2}\left[\left(\overline{\mathrm{x}}_{2}-\overline{\mathrm{y}}_{2}\right)^{2}+\left(\overline{\mathrm{x}}_{3}-\overline{\mathrm{y}}_{3}\right)^{2}\right]}
$$

in which $\bar{x}_{1}$ and $\bar{y}_{1}$ are the projected components of the vectors $\mathrm{x}$ and $\mathrm{y}$ on the Mach number vector $M$, respectively, at the observer time. The components $\overline{\mathrm{x}}_{2}$, $\overline{\mathrm{x}}_{3}$ and $\overline{\mathrm{y}}_{2}, \overline{\mathrm{y}}_{3}$ are the transverse components to $\overline{\mathrm{x}}_{1}$ and $\overline{\mathrm{y}}_{1}$, respectively.

The Kirchhoff surface integrations are preformed assuming that the integrand in Eqn. 12 is constant over a Kirchhoff surface element, and the time is discretized in the same manner as in the flow solver. The acoustic pressure required by Eqn. 12 in the vicinity of the Kirchhoff surface is obtained from the flow solver by subtracting the mean pressure from the instantaneous pressure. In most cases the time increment of the flow solver, $\Delta t$, from one time step to the next is sufficiently small to calculate the time derivative of the acoustic pressure on the Kirchhoff surface using a first-order backward difference. Then,

$$
\left.\frac{\partial \mathrm{p}_{\mathrm{e}}^{\prime}}{\partial \tau}\right|_{\tau^{*}}=\frac{\mathrm{p}_{\mathrm{e}}^{\mathrm{n}^{*}}-\mathrm{p}_{\mathrm{e}}^{\mathrm{n}^{*}-1}}{\Delta \mathrm{t}}
$$

where the superscript $\mathrm{n}^{*}$ is the time level such that, when multiplied by $\Delta t$, it gives a discrete time that is the closest to the emission time $\tau_{\mathrm{e}}^{*}$. This is only an approximation provided that $\Delta t$ is sufficiently small. Hence we do not perform interpolations for the exact emission time data. The gradient of the acoustic pressure is calculated using the same finite difference stencils as the flow solver. At this point the observer time is considered the same as the flow solver. Hence, for the discrete acoustic pressure at the observer point and time, we can write

$$
\mathrm{p}^{\prime}[\mathrm{x}, \mathrm{n} \Delta \mathrm{t}]=\frac{1}{4 \pi} \sum_{\mathrm{e}=1}^{\mathrm{NE}} \mathrm{P}_{\mathrm{e}}\left\{\left[\mathrm{n}-\operatorname{int}\left(\mathrm{R}_{\mathrm{e}}\right) / \mathrm{c}_{\infty}\right] \Delta \mathrm{t}\right\} \Delta \mathrm{S}_{\mathrm{e}}
$$

where the operator int( ) rounds the real argument to the closest integer. The time level $\left(n-\operatorname{int}\left(R_{e}\right) / c_{\infty}\right)$ in Eqn. 20 is identical to the time level $n^{*}$ that appears in Eqn. 19. Implementation on a distributed computing environment is performed by letting each processor identify that part of the Kirchhoff surface which is located in its computational domain. Before the time integration process begins, the lag time associated with each element is calculated and a global minimum is communicated to each processor. This marks the starting point of the acoustic pressure storage array on all processors. During the iterative process, each processor sums the contributions of acoustic pressure from the elements in its computational domain. After the simulation, the acoustic pressure arrays from all processors are summed up to get the final response in the farfield. 


\subsection{Source Terms}

The source terms that simulate the effect of the sound generating mechanisms involving rotor-stator interactions are derived from infinite duct theory. Duct acoustic modes are classified as $(\mathrm{m}, \mu)$ representing the circumferential and radial modes. The circumferential mode is related to the number of stator vanes and rotor blades through the relation

$$
\mathrm{m}=\mathrm{n} \mathrm{B}+\mathrm{sV} \quad \mathrm{s}=\ldots-2,-1,0,1,2 \ldots
$$

The acoustic pressure in an infinite cylinder as a function of $(\mathrm{r}, \theta, \mathrm{x})$ is given as

$$
\begin{array}{r}
\hat{\mathrm{p}}_{\mathrm{nm} \mu}(\mathrm{x}, \mathrm{r}, \theta,)=\mathrm{A}_{\mathrm{nm} \mu} \mathrm{J}_{\mathrm{m}}\left(\mathrm{k}_{\mathrm{m} \mu} \mathrm{r}\right) \\
\exp \left[\mathrm{i}\left(\mathrm{k}_{\mathrm{x}, \mathrm{m} \mu} \mathrm{x}+\mathrm{m} \theta+\phi_{\mathrm{nm} \mu}\right)\right]
\end{array}
$$

A summation over all the eigensolutions corresponding to all active modes gives us a representative source term that can be specified at the fan face

$$
\mathrm{p}_{\mathrm{f}}^{\prime}(\mathrm{x}, \mathrm{r}, \theta, \mathrm{t})=\Re\left(\sum_{\mathrm{n}=1 \mathrm{~m}=-\infty}^{\infty} \sum_{\mu=0}^{\infty} \sum_{\mathrm{p} m \mu} \mathrm{e}^{-\mathrm{i} \omega \mathrm{nt}}\right)
$$

The axial wave number $\mathrm{k}_{\mathrm{x}, \mathrm{m} \mu}$ is given by

$$
\mathrm{k}_{\mathrm{x}, \mathrm{m} \mu}=\frac{-\mathrm{M}_{\mathrm{f}} \mathrm{k}-\sqrt{\mathrm{k}^{2}-\left(1-\mathrm{M}_{\mathrm{f}}^{2}\right)} \mathrm{k}_{\mathrm{m}, \mu}^{2}}{1-\mathrm{M}_{\mathrm{f}}^{2}}
$$

And the axial wavelength $\lambda_{\mathrm{a}}=\frac{2 \pi}{\mathrm{k}_{\mathrm{x}, \mathrm{m} \mu}}$ with the sign of $\mathrm{k}$ determining an upstream or downstream travelling. The cut-off ratio of a particular mode $(m, \mu)$ is given by

$$
\xi_{\mathrm{m} \mu}=\frac{\mathrm{nBM}_{\mathrm{T}}}{\mathrm{r}_{\mathrm{f}} \mathrm{k}_{\mathrm{m} \mu} \sqrt{1-\mathrm{M}_{\mathrm{f}}^{2}}}
$$

where $\mathrm{M}_{\mathrm{T}}$ is the rotor tip speed.

\subsection{Grid Topology}

Among the different grid options, the use of an $\mathrm{O}-\mathrm{H}$ grid type seems attractive for engine calculations since it is typically used in steady state computations. However this grid typology has a singularity at the highlite of the nacelle requiring clustering in the region (as shown in Fig. 3). Furthermore, the clustering is carried all the way to the boundaries and it creates difficulties in resolving higher order schemes like those utilized in wave propagation problems. Furthermore, the grids need to be orthogonal to each other, especially close to the centerline, for the sake of geometrical conservation during discretization of the equations. For engine inlet configurations, grids generated (Fig. 4) by conformal mappings (Ref. 13) have provided smooth orthogonal grids. Based on this topology, we generated grids (Fig. 5) for the entire inlet/exhaust configuration. Although, these grids are orthogonal throughout, they exhibit one drawback. There is an interchange in the marching direction at two points as shown in Fig. 5 on the cowl surface. These crossover points have difficulties with a higher order scheme thereby limiting its utility for our purposes. In this paper we utilize a wrap around O-type grid starting at the fan face and terminating at one exhaust/stator plane. This type of grid traces a wavefront and naturally coarsens close to the farfield boundary and provides fine resolution in the region of interest.

\subsection{DISCUSSION OF RESULTS}

\subsection{Radiation from a Dipole in a Cylinder}

In order to test our approach for entire engine calculations and evaluate the appropriateness of the grid topology in use, we compare our results for radiation from a dipole in a cylinder, with the boundary element method TBIEM3D. This is a close representation of the radiation problem from an engine. The TBIEM3D [12] code is a frequency domain code. This code can simulate radiation from cylindrical shaped containers (with both ends open) including forward flight effects. The noise due to the fan blades is modeled using rotating or non-rotating dipole sources. This is a linearized code with limited cowling shape options, but is extremely useful for validation and trade studies.

The problem solved here consists of a dipole placed at the center (on the axis of symmetry). The boundary element method utilizes an infinitely thin shell to represent the cylinder. In our approach, the shell has a finite thickness and the edges are rounded to alleviate problems in gridding. A wrap-around O-type grid is used with the grid being cyclic in the primary direction. As is seen in Figure 6, the cylinder is located at $-15<x<15$ and $\mathrm{y}=15$. The dipole can be represented as a combination of two monopoles with the phase shift of 180 degrees or as a source term [14] in the momentum equation given by

$$
S=A \rho_{\infty} \exp \left\{\frac{-3}{(\Delta \omega \Delta x)^{2}} r^{2} \sin \omega t\right\}
$$

where $\Delta \omega$ is the radius of Gaussian pulse in terms of number of cells.

The source term is valid everywhere inside the cylinder and is zero outside. For these computations a wavenumber of 267 radians/sec is utilized and no mass flow is specified. Figure 6 shows the instantaneous pressure contours of the TBIEM3D compared to the Euler solution. The two solutions indicate close agreement, and the Euler solution 
seems to capture the interference effects behind the cylinder due to waves emanating from both the aft and front ends.

There is a slight discrepancy in the solutions due to the rounding of the edges. This is better seen in Figure 7 that depicts the comparison of sound pressure levels. As we move away from the cylinder, axially, a phase shift error starts to dominate the Euler solution. This is due to poor resolution that is an artifact of grid coarsening inherent in grid topologies of this nature.

\subsection{Radiation from Engine}

Our next set of results pertains to computations regarding a realistic ducted fan and exhaust system. The engine contains 18 rotor blades and a set of 42 stator vanes. The operating flow regime for this engine dictates a freestream Mach No. of 0.2 and the fan rotates at an RPM of $5210 \mathrm{rev} / \mathrm{min}$. This engine has a highlight radius of 0.28 meters and a centerbody that varies significantly throughout the length of the cowl and extends beyond, arcing towards the centerline as it reaches the compressor exhaust. The rotor blades extend from $-0.041 \mathrm{~m}$ to $0.047 \mathrm{~m}$ and the stator blades are located between $0.1478 \mathrm{~m}$ and $0.193 \mathrm{~m}$. For the purpose of our calculations, we have taken a fixed rotor and stator plane that is obtained by arithmetical averaging. For our purposes, these represent the inlet and exhaust planes of our calculations. Furthermore, the compressor exhaust is eliminated by carrying the aft end of the centerbody all the way to the axis of symmetry.

\subsection{Radiation of the $(0,0)+(0,1)$ Modes with No Mass Flow and without a Centerbody} For our first calculations we removed the centerbody, so that our system now approximates a cylindrical duct with a cowl. For the purpose of generating the zeroth circumferential mode at the blade passage frequency, we assume that the number of stator vanes is equal to the number of rotor blades. The source consists of a combination of the $(0,0)$ and $(0,1)$ modes, each having a $3 \mathrm{kPa}$ amplitude. From past experience [6] it has been found that this high source amplitude does not create any nonlinearities in the absence of mass flow. Both at the exhaust and inlet planes momentum source terms are specified based on the linearized momentum equations and the acoustic pressure obtained from cylindrical duct theory.

$$
\begin{gathered}
\left(\rho \hat{u}_{1}\right)_{\mathrm{nm} \mu}=\rho_{\infty} \Re\left[\frac{\mathrm{k}_{\mathrm{x}, \mathrm{m} \mu}}{\rho_{\infty} \mathrm{n} \omega} \mathrm{p}_{\mathrm{nm} \mu}^{\prime}\right] \\
\left(\rho \hat{\mathrm{u}}_{2}\right)_{\mathrm{nm} \mu}=\rho_{\infty} \Re\left[\frac{\mathrm{k}_{\mathrm{m} \mu}}{\rho_{\infty} \mathrm{n} \omega} \frac{\mathrm{J}_{\mathrm{m}}^{\prime}\left(\mathrm{k}_{\mathrm{m} \mu} \mathrm{r}\right)}{\mathrm{J}_{\mathrm{m}}\left(\mathrm{k}_{\mathrm{m} \mu} \mathrm{r}\right)} \mathrm{p}_{\mathrm{nm} \mu}^{\prime}\right]
\end{gathered}
$$

$$
\begin{aligned}
& \left(\rho \hat{u}_{3}\right)_{n m \mu}=\rho_{\infty} \Re\left[\frac{m}{\rho_{\infty} n \omega r} p_{n m \mu}^{\prime}\right] \\
& \left(\rho u_{1}\right)(x, r, \theta, t)=\sum_{n=1}^{\infty} \sum_{m} \sum_{u=0}^{\infty}\left(\rho \hat{u}_{1}\right)_{n m \mu}, \quad i=1,2,3
\end{aligned}
$$

where the value of $\mathrm{P}^{\prime}{ }_{\mathrm{nm} \mu}$ can be found from Eqn. 22. Based on cylindrical duct theory, the cut-off ratios for the two modes are $\xi_{m \mu}(0,0)=\infty$ and $\xi_{m \mu}(0,1)=2.109$ indicating both modes to be cut-on. As is seen from Figure 8 the waves are well resolved almost until the farfield boundary and the interference patterns closely resemble those obtained for the dipole in the cylinder. The sound pressure level directivity patterns also closely resemble those obtained for the cylindrical duct with the dipole. The normalized SPL levels are shown in Figure 9 and indicate multiple lobes in both the aft and the front ends. The maximum levels occur close to the inlet axis.

\subsection{Radiation of the $(0,0)+(0,1)$ Modes with No Mass Flow and with a Centerbody}

In the next set of calculations we include the centerbody in our inlet/exhaust system. Again, we assume parity in the number of stator vanes and rotor blades in order to generate the zeroth circumferential mode at the blade passage frequency. The $(0,0)$ and $(0,1)$ modes are excited at an amplitude of $3 \mathrm{kPa}$ and the boundary conditions are imposed in much the same manner as the previous case. However, in this case Bessel functions of the second order are utilized to evaluate the acoustic pressure source terms since the exhaust and inlet planes are now representative of annular ducts. As a consequence, the cut-off ratios for the exhaust plane are $\xi_{m \mu}(0,0)=1.3$ and $\xi_{m \mu}(0,1)=0.65$ and for the inlet plane are $\xi_{m \mu}(0,0)=1.62$ and $\xi_{m \mu}(0,1)=0.83$. Both the $(0,1)$ modes are now cut-off and this explains the rapid dampening of waves as they travel outwards. As is seen in Figure 10 this effect is more pronounced at the aft end, where the cut-off ratios are lower compared to the front end.

\subsection{Radiation with Mass Flow and Centerbody}

In this section, we present results with mass flow. The steady state solution was obtained with mass flow at the inlet face being $17.8 \mathrm{~kg} / \mathrm{sec}$ and the mass flow specified at the state of exhaust phase is increased by $10 \%$ $(20.05 \mathrm{~kg} / \mathrm{sec}$.). This simulates the effect of an engine and the velocity at the backend is matched to that observed experimentally. Mach number contours of the steady state solution are shown in Figure 11. Multigrid acceleration was used, although convergence dropped only four orders of magnitude. This may be due to the unsteadiness inherent in the shear layer at the aft end. A source term defining a plane wave excitation at the blade passage frequency was turned on at the stator and rotor planes and the acoustic pressures are shown in Figure 12. A distinct was pattern is seen both at the fore and the aft end. 
Further investigation is being carried out to improve the characteristic boundary conditions in the near fields as acoustic pressure levels indicate reflections in the domain.

\subsection{SUMMARY AND FUTURE WORK}

In this paper we have extended the hybrid, time domain ducted for noise prediction capability developed for engine inlets by Ozyoruk and Long to entire engine configurations. An MPI based parallel version of the code suitable to distributed computing platforms has been developed. The code solves the 3-D nonlinear Euler/Navier-Stokes equations and is coupled to a Kirchhoff method for farfield predictions. The implementation of multigrid is essential or convergence acceleration and accuracy purposes. Comparisons with frequency domain codes show good agreement for radiation from a dipole in a cylinder. Furthermore, simulations of radiation from a full engine configurations have been demonstrated. The results show qualitative trends that closely approximate the patterns obtained for one dipole in the cylinder that are considered representative of engine radiation patterns.

Most simulations involved the zeroth circumferential mode. Currently, analysis of the spinning modes are underway. In particular, the sixth circumferential mode is being investigated for the engine configuration shown in this paper. Efforts are also underway to use a soft wall boundary condition to simulate the effect of engine liners.

\subsection{ACKNOWLEDGEMENTS}

The authors wish to acknowledge the technical support and insightful comments provided by Rumsey, R.T. Biedron and F. Farassat of the NASA Langley Research Center. The lead author would like to thank Ms. Katherine Young for her help in preparing the paper.

\subsection{REFERENCES}

[1] Tyler, J.M., and Sofrin, T.G., "Axial Flow Compressor Noise Studies," SAE transactions, Vol.70, 1962, pp 309-332.

[2] Ahuja, V., Deshpande, M., Feng, J., Wang, L., and Merkle, C.L., "Practical Applications of Parallel Processing in Computational Fluid Dynamics," Proceedings of $13^{\text {th }}$ AIAA CFD Conference, Snowmass Village, CO, June 29-July 2, 1997.

[3] Rumsey, C.L., Biedron, R.T., Farassat, F. and Spence, P.L., "Ducted Fan Engine Acoustic Predictions Using a Navier Stokes Code," Journal of Sound and Vibration, Vol. 213, No. 4, June 1998, pp 643-664.

[4] Everson, W., "A Finite Element Formulation for Aft Fan Duct Radiation," AIAA Paper No. 97-1648.
[5] Myers, M.K., "Boundary Integral Formulations for Ducted Fan Radiation Calculations," CEAS-AIAA Paper No. 95-076, June 1995.

[6] Ozyoruk, Y., and Long, L.N., "Computation of Sound Radiating from Engine Inlets," AIAA Journal, Vol.34, No. 5, May 1996.

[7] Farassat, F. amd Myers, M.K., "Extension of Kirchoff's Formula for Radiation from Moving Surfaces," Journal of Sound and Vibration, Vol. 123, June 1988, pp451-460.

[8] Ozyoruk, Y., and Long, L.N., "A New Efficient Algorithm for Computational Aeroacoustics on Massively Parallel Computers," Journal of Computational Physics, Vol. 125, No. 1, pp. 135149, April, 1996.

[9] Jameson, A., Schmidt, W., and Turkel, E., "Multigrid Algorithms for Compressible Flow Calculations. In Lecture Notes in Mathematics, Number 1228 in Multigrid Methods II" Proceedings of the $2^{\text {nd }}$ European Conference on Multigrid Methods, October 1985, pp. 66-201, Spring Valley, 1986.

[10] Ozyoruk, Y., and Long, L.N., "Multigrid Acceleration of a High Resolution Aeroacoustic Scheme," AIAA Journal, Vol. 35, No. 3, pp. 428433, March, 1997.

[11] Jameson, A., "Numerical Solutions of the Euler Equations by Finite Scheme methods using RungeKutta Time-stepping Schemes," AIAA Paper No. 811259, 1981.

[12] Dunn, M. H., “TBIEM3D A Computer program for Predicting Ducted Fan Engine Noise," Version 1.1, NASA CR-97-206232, Sept., 1997.

[13] Ives, D.c. and Menor, W.A., "Grid Generation for Inlet-Centerbody Configurations using Conformal mapping and Stretching," AIAA Paper No. 81-0097, 1981.

[14] Pierce, A.D., "Linear Acoustics," Encyclopedia of Applied Physics, Vol. 1, pp. 137-182, 1991.

[15] Lyrintzis, A.S., "The Use of Kirchoff's Method in Computational Aeroacoustics," FED-Vol. 147, Computational Aero and Hydro Acoustics. 


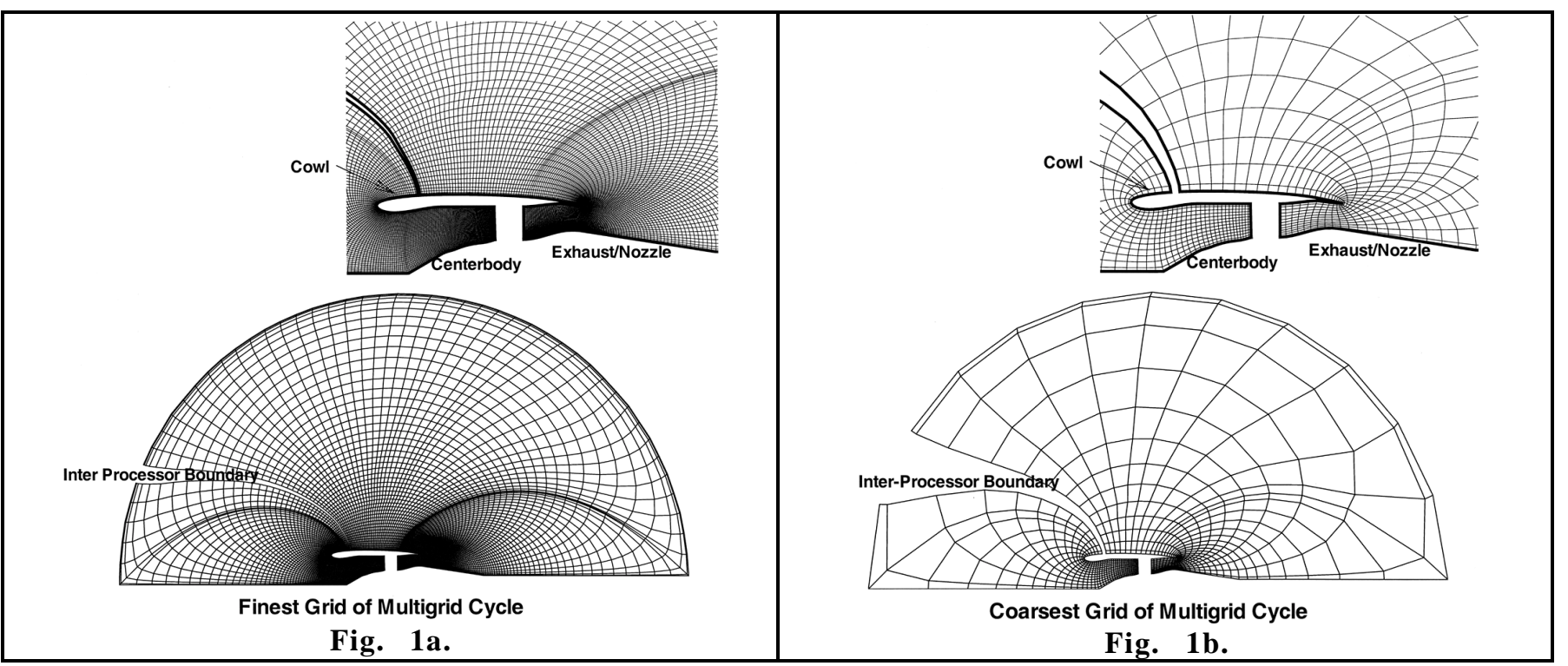

Fig. 1. Fine and coarsest grids of the multigrid cycle.

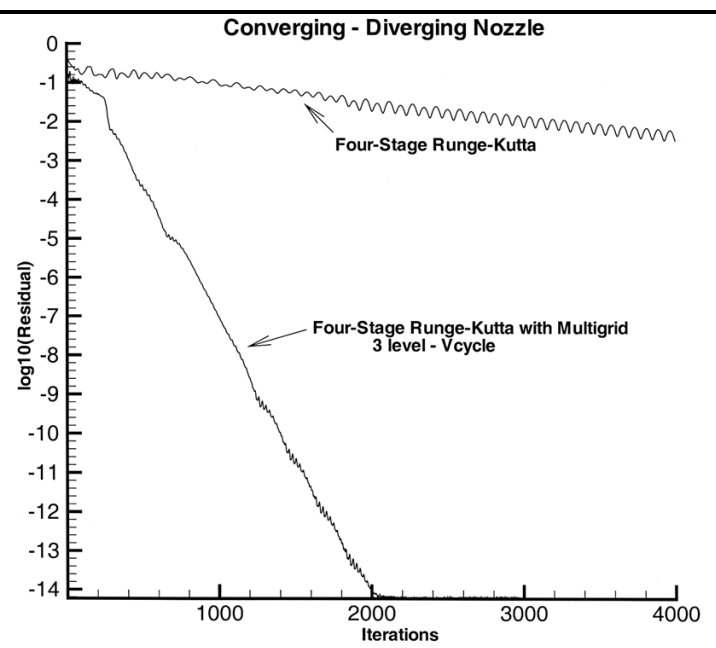

Fig. 2. Convergence comparisons with and without multigrid for a converging-diverging nozzle.

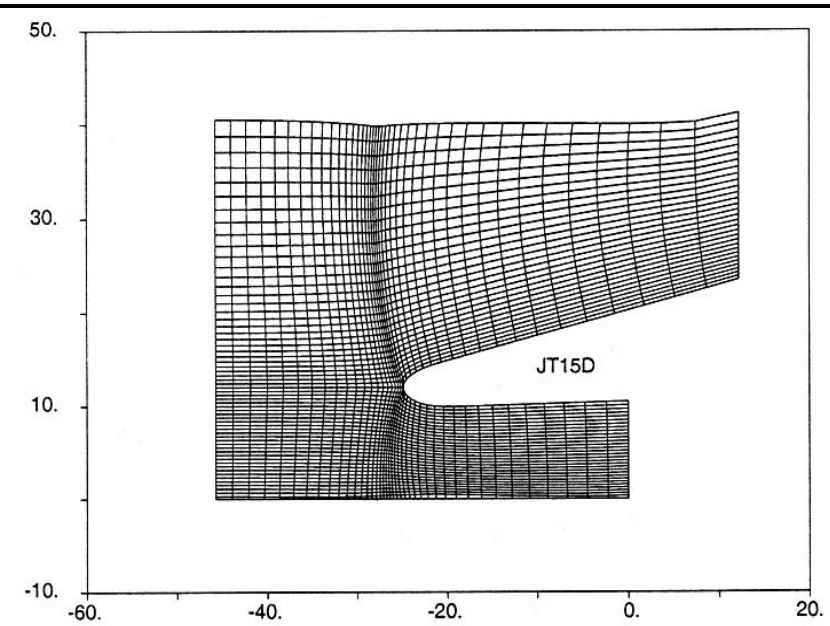

Fig. 3. H-grid used in steady state computations of an engine inlet.

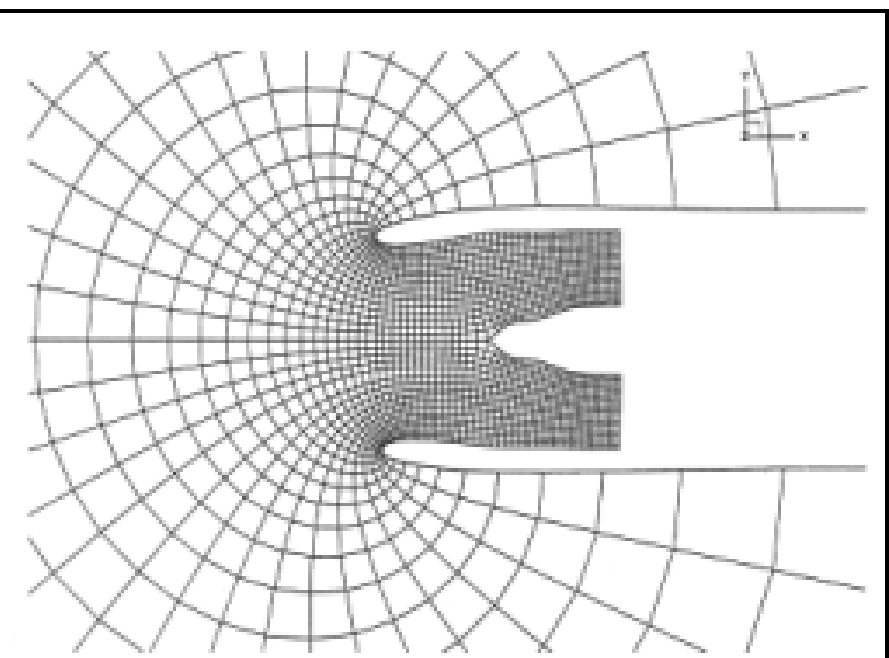

Fig 4. Conformal mapping based grid used for solving radiation problems from inlet.

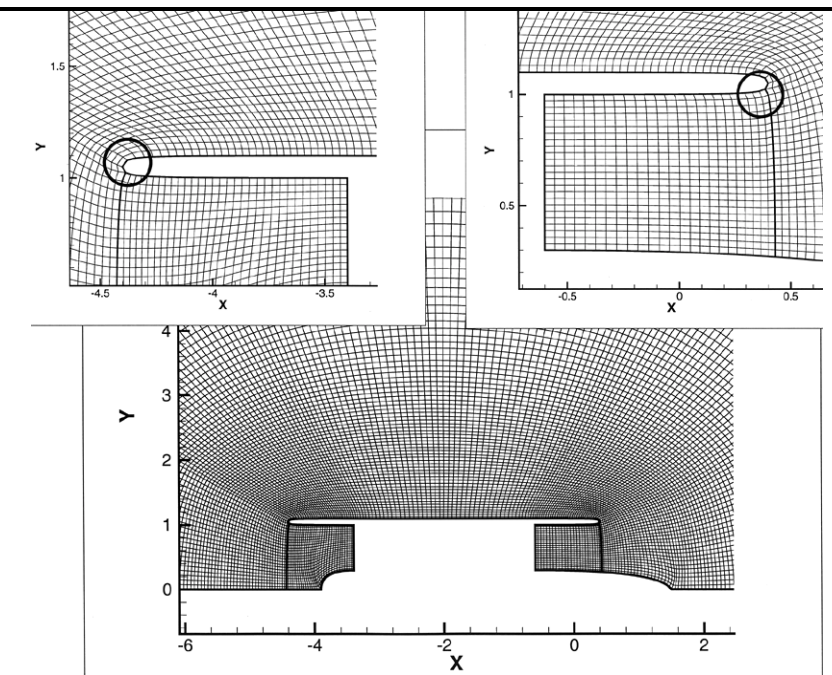

Fig. 5. Grid for entire engine-like geometry extending the topology of conformal mapping based grids. 

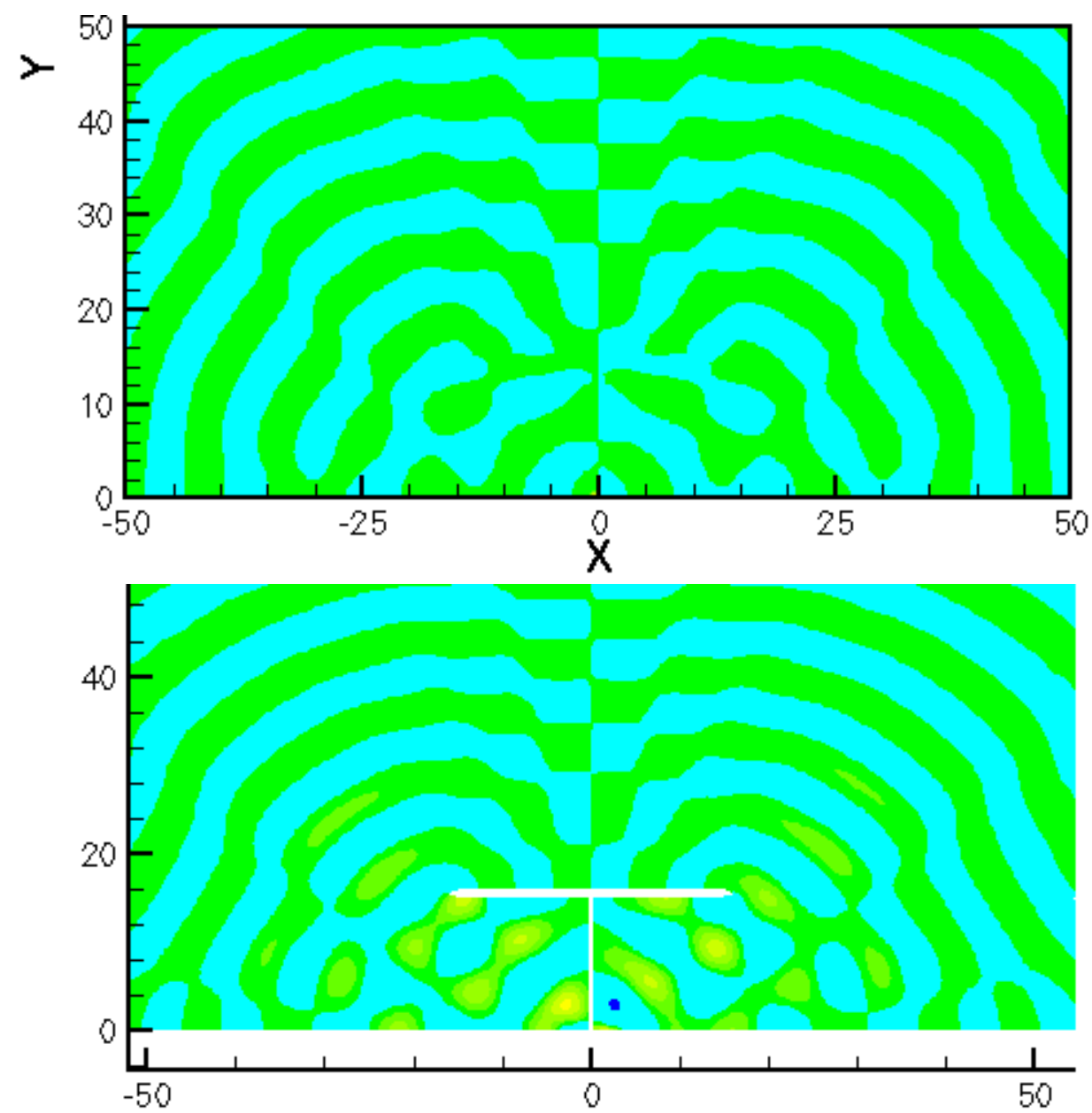

Fig. 6. Radiation patterns of acoustic pressure for a dipole in a cylinder. The top figure is TBIEM3D solution. The bottom figure is Euler calculation.

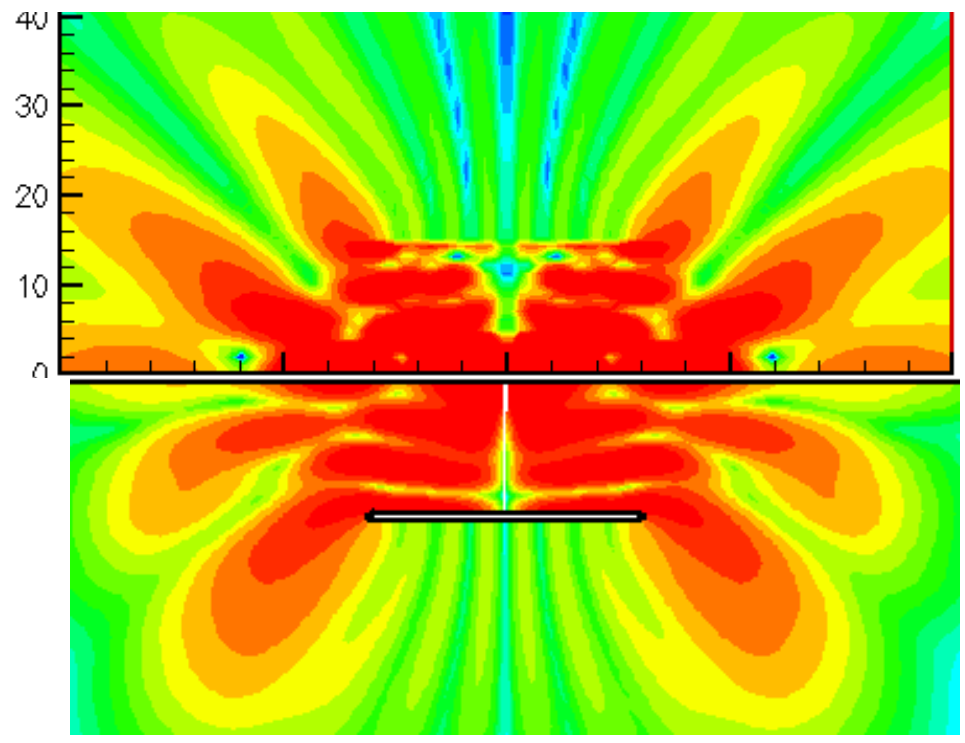

Fig. 7. SPL contours for a dipole in a cylinder.

The top figure is TBIEM3D solution. The bottom figure is Euler calculation. 


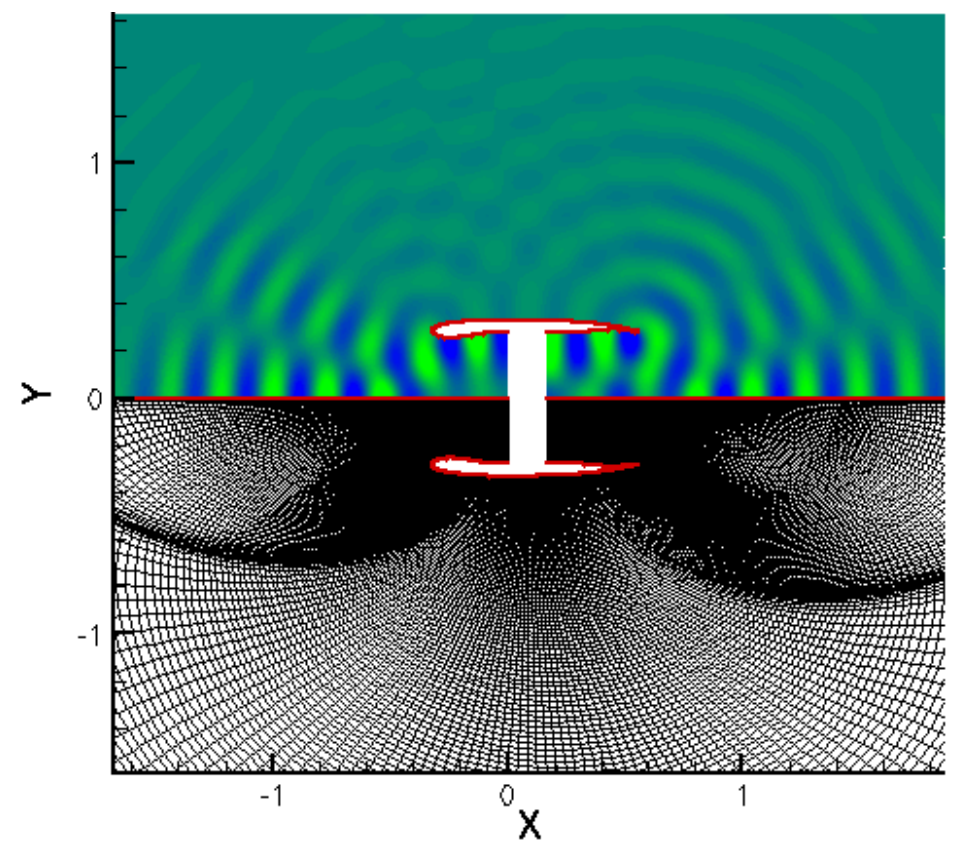

Fig. 8. Radiation from engine without centerbody for $(0,0=(0,1)$ modes and no flow.

Patterns range from $-300 \mathrm{~Pa}$ to $300 \mathrm{~Pa}$. A section of the grid is shown in the bottom half of the figure.

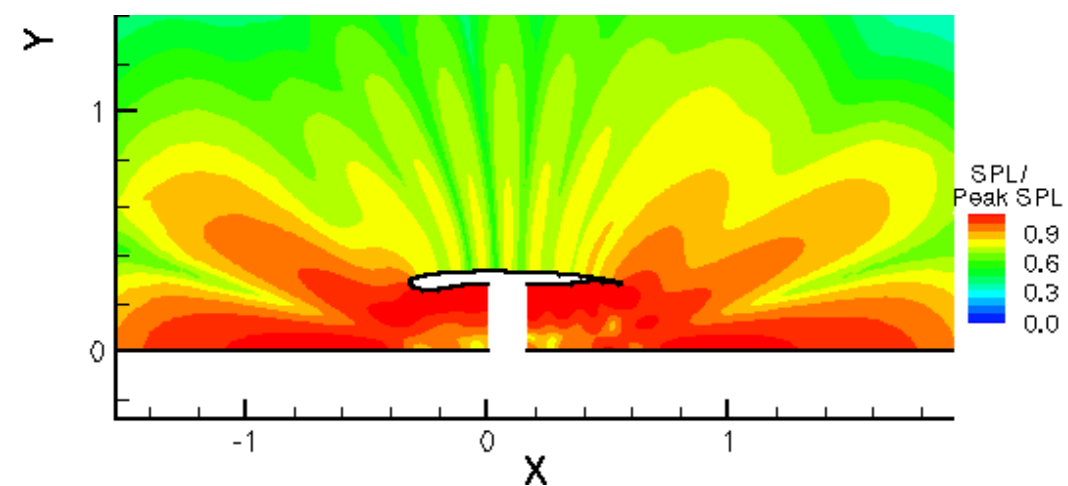

Fig. 9. SPL levels for radiation from engine without centerbody with $(0,0)=(0,1)$ modes.

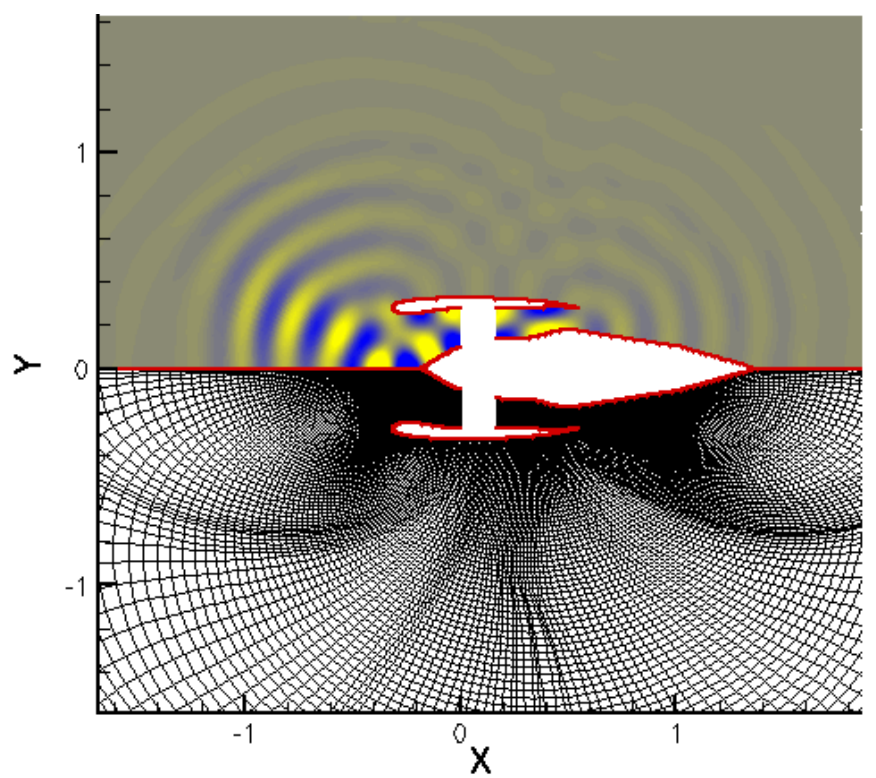

Fig. 10. Radiation from engine with centerbody with $(0,0)+(0,1)$ modes and no flow. Acoustic pressure patterns range from $-300 \mathrm{~Pa}$ to $300 \mathrm{~Pa}$. The bottom half showing grid used for the calculation. 


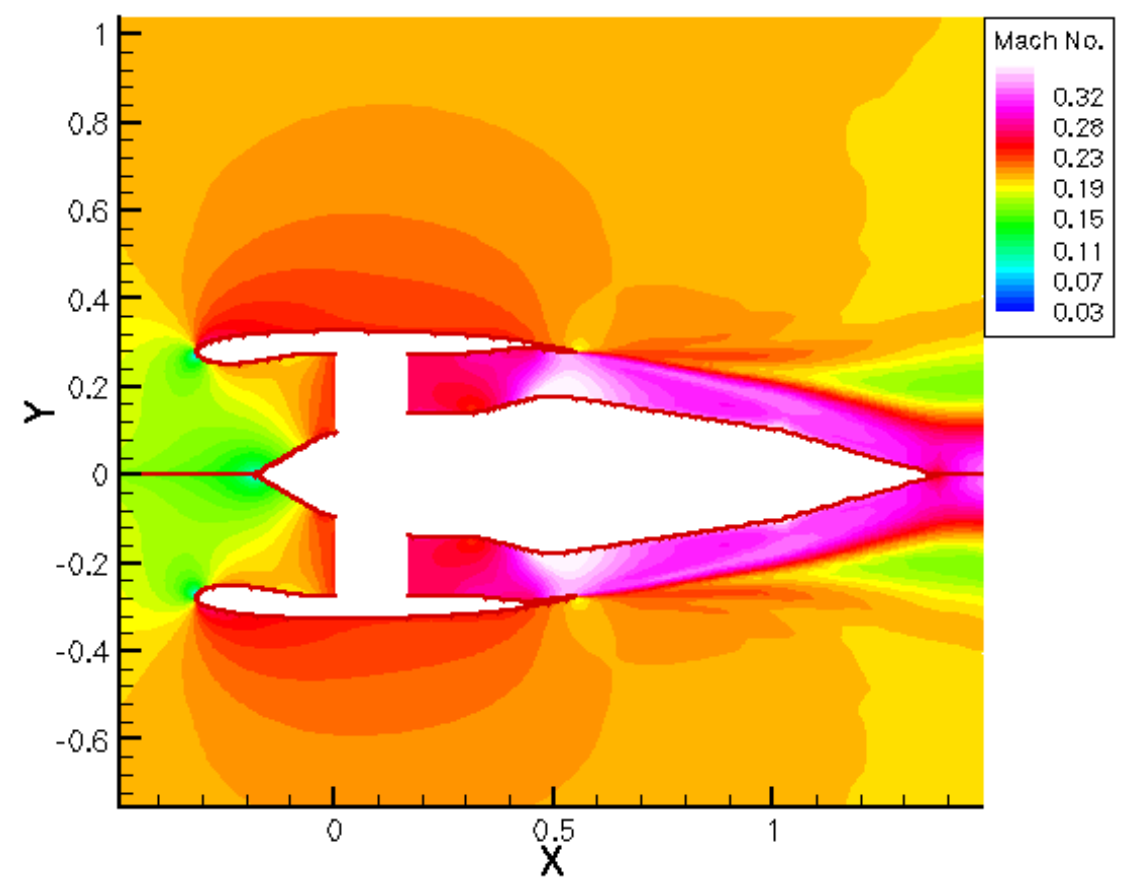

Fig. 11. Mach number contours for the steady state solution of engine with centerbody.

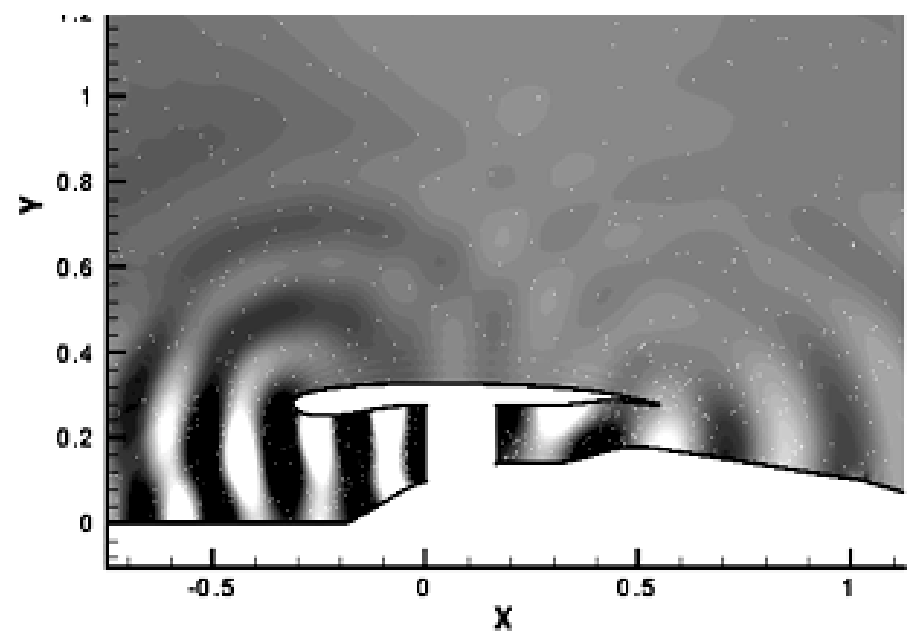

Fig. 12. Acoustic pressure patterns from engine with mean flow and plane wave excitation. Levels range from $-400 \mathrm{~Pa}$ to $400 \mathrm{~Pa}$. 